\title{
New highlight in breast cancer development: the key role of hepcidin and iron metabolism
}

\author{
Manuel Scimeca ${ }^{1,2}$, Elena Bonanno, \\ ${ }^{1}$ Department of Biomedicine and Prevention, University of Rome "Tor Vergata”, Rome, Italy; ${ }^{2}$ IRCCS San Raffaele, Rome, Italy; ${ }^{3}$ Department of \\ Experimental Medicine and Surgery, University “Tor Vergata”, Rome, Italy; "'Diagnostica Medica” and "Villa dei Platani”, Avellino, Italy \\ Correspondence to: Elena Bonanno, MD, PhD. Associate Professor of Pathology, Department of Experimental Medicine and Surgery, University of \\ Rome Tor Vergata, Via Montpellier, 1, Rome 00133, Italy. Email: elena.bonanno@uniroma2.it. \\ Comment on: Blanchette-Farra N, Kita D, Konstorum A, et al. Contribution of three-dimensional architecture and tumor-associated fibroblasts to \\ hepcidin regulation in breast cancer. Oncogene 2018;37:4013-32.
}

Submitted Sep 26, 2018. Accepted for publication Oct 10, 2018.

doi: $10.21037 / \mathrm{atm} .2018 .10 .30$

View this article at: http://dx.doi.org/10.21037/atm.2018.10.30

Breast cancer is one of the most frequent neoplasms diagnosed every year in the world. Among women, more of $30 \%$ of all cancer lesions are breast cancer. In the United States only, $12.5 \%$ of women will be affected by invasive breast cancer during their lifetime (1). In 2017, approximately 252,000 new infiltrating breast tumors were detected in the United States (2). In spite of successful resection or chemo - and radiotherapy, tumor relapse and metastasis are the primary causes of poor prognosis among patients with breast cancer. In detail, about $10 \%$ of all breast cancer patients without clinical proof of metastatic lesions at the first diagnosis will develop a first relapse within five years of their primary diagnosis (3). Moreover, $65-75 \%$ of patients with breast neoplasms will experience metastases, especially in the bone (3). These complications significantly affect the patient's quality of life also contributing to the cost of care (4). Thus, the identification of molecular mechanisms involved in breast cancer occurrence and development represents one of the most important goals of the biomedical research. In line with these considerations, Blanchette-Farra and collaborators recently proposed a new paradigm for tumor-mediated control of iron via hepcidin by tumor architecture and the breast tumor microenvironment (5). Authors used 3D cell culture method by investigating breast cancer spheroids derived from both MCF-7 and MCF-10a cell lines and primary cell culture from breast cancer patients to study the possible role of cancer-associated fibroblasts in the regulation of breast tumor pathways by iron and hepcidin (5). Hepcidin is a peptide hormone that binds to ferroportin and elicits its degradation, playing a not redundant role in breast disease by increasing iron cytoplasmic concentration (6).

In agreement of this, the study of Blanchette-Farra et al. showed that the metabolism of hepcidin in breast cancer was driven by several molecules of the TGF-B super-family, as bone morphogenetic proteins (BMPs) and growth differentiation factor-15 (GDF-15) (5). More in detail, expression of GDF-15 from patient's samples were significantly associated with levels of hepcidin at both the mRNA and protein level, thus suggesting a role for GDF-15 in the regulation of hepcidin in human breast cancers (5). As concern the correlation between hepcidin and BMPs expression, authors observed that, BMPs, particularly BMP6, were important mediators for hepcidin synthesis in breast cancer cells, studied both in 2D and in $3 \mathrm{D}$ cell culture systems (5). In addition, inclusion of tumorassociated fibroblasts in breast cancer spheroids further induces hepcidin by fibroblast-dependent secretion of IL-6, one of the most important pro-inflammatory cytokine (5).

However, a very important message from this study is that a dysregulation in the spatial configuration of cancer cells is enough to modify responses to extracellular stimuli and promote several pathways of hepcidin induction. Thus, even though cells cultured in $2 \mathrm{D}$ showed receptors able to make them responsive to IL-6, hepcidin synthesis was not induced by treatment with exogenous IL-6 in cells 
grown in 2D. On the other hand, breast cancer cells grown in $3 \mathrm{D}$ culture conditions induced hepcidin when exposed to IL-6. This evidence points out the relevance of spatial organization in the stimulation of tumor signaling pathways, including those that control iron metabolism. From clinical point of view, data of Blanchette-Farra are strengthen by a large cohort study of Pinnix et al. in which has been demonstrated that the combined expression profile of ferroportin and hepcidin is a powerful predictor of survival after mastectomy for women with breast cancer (7).

This new and interesting data acquire further relevance if compared with the more recent discoveries in the field of breast cancer metastasis to bone. In particular, Scimeca et al. have recently described a new breast cancer cell type showing morphological, molecular and functional characteristics of osteoblasts $(8,9)$. These cells originate in breast environment under the stimulation of several osteoblast differentiation factors expressed by cancer cells, inflammatory cells and peri-tumoral fibroblasts, i.e., BMPs (10) and PTX3 $(11,12)$. In addition, has been demonstrated that the presence of BOLCs in primary lesions can predict the formation of bone metastasis lesions within 5 years from the diagnosis of breast primary carcinoma (13). It is important to note that the hepcidin and iron metabolism are key elements for the differentiation and activity of real osteoblasts and thus for bone metabolism.

In a study of $\mathrm{Li}$ and colleagues, authors showed that hepcidin is able to increase the cytoplasmic levels of calcium in osteoblast cells, demonstrating that this effect was more pronounced if osteoblasts were exposed to increasing concentrations of iron (14). To further characterize this phenomenon, authors used a L-type calcium channel blocker (nimodipine) and a ryanodine receptor antagonist (dantrolene) to inhibit the calcium flux through the sarcoendoplasmic reticulum of osteoblasts (14). The absence of significant modification in cytoplasmic calcium levels in human osteoblasts exposed to these molecules suggested a precise relationship between hepcidin expression and calcium homeostasis. In fact, this mechanistic relationship seems to be mediated by the release of calcium from endoplasmic reticulum, which is triggered by calcium influx (14). In vitro studies further described the possible role of hepcidin and iron metabolism in osteoblasts differentiation displaying that hepcidin expression may decrease rate of apoptosis and enhance the formation of calcium nodules. Therefore, hepcidin protected bone quality by both reducing the osteoblast apoptosis and promoting the physiological process of mineralization. Moreover, has been shown that hepcidin induced the expression of essential molecules involved in bone mineralization such as collagen1 (COL1), BMP2 and osteoprotegerin (OPG) thus promoting osteoblast differentiation and function (15). Starting from these considerations is possible to speculate about the connection between hepcidin expression, iron metabolism, breast development, tumor environment and BOLCs generations. Indeed, all these elements can play an important role in the formation of bone metastatic lesions by BOLCs. Specifically, iron metabolisms and hepcidin expression can orchestrate the early phases of BOLCs formation by regulating the BMPs homeostasis. In addition, the presence of high amount of iron may enhance breast tumor initiation, growth and metastases by promoting redox cycling of estrogen metabolites (16). In agreement with this, BOLCs are mainly detected in the breast lesions characterized by both expression of estrogen receptors and propensity to form bone metastasis i.e., luminal A subtype $(9,13)$. Modifications in iron homeostasis in cells of breast tumor microenvironment as macrophages and fibroblasts may also foster breast to bone metastatization. Thus, expression of genes involved in iron metabolism could be predictive of breast cancer prognosis. Iron chelators and other approaches conceived to limit iron may acquire therapeutic significance in breast cancer especially for what concern the development of bone metastasis (16). The dependence of breast cancer on iron presents rich opportunities for improved prognostic evaluation and therapeutic intervention. Several in vivo and ex vivo techniques could be used to study the iron metabolism in breast. In particular, distribution of iron in breast environment may be investigated exploiting the potential of nuclear medicine. In fact, currently, numerous radiotracers as ${ }^{52} \mathrm{Fe}$-citrate are able to track iron particles in peculiar human pathologies such as Wilson's disease (17). Moreover, there is an increase of iron in the brain with aging and increased iron levels were observed along with deposits of senile plaque in Alzheimer patients (18). Similarly, this approach could be proposed for the screening of breast cancer patients in order to identify the lesions with high probability to develop bone lesions. Also, accumulation of iron in breast can be studied in bioptic samples by using energy dispersive X-ray (EDX) microanalysis (19). EDX microanalysis is a technique that allows the analysis of elemental composition of a very small fragment of tissue. Its performance is based on the generation of characteristic $\mathrm{X}$-rays that reveals the presence of elements in the specimens subjected to electron microscopy analysis (19). 
EDX is frequently used in the study of drugs, such as in the study of drugs delivery in which the EDX is an important tool to identify nanoparticles (19). It is also used for the investigation of environmental pollution and for detection of metal accumulated in the normal and pathological tissues $(19,20)$. The application of EDX can provide the subcellular localization of iron in breast cancer cells allowing to shed light the possible accumulation of this metal in the BOLCs.

In conclusion, despite many recent advances, breast cancer remains a clinical challenge. The identification of specific pathways involved in breast cancer occurrence and progression require a multidisciplinary approach that involved several biomedical disciplines as anatomic pathology and nuclear medicine (21). There are several interesting new techniques on the horizon that could provide a notable improvement in breast cancer detection by analysis of hepcidin and iron metabolism. The future introduction of these techniques in the diagnosis of breast cancer could great improve the management of breast cancer patients providing to oncologist new options for both diagnosis and therapy.

\section{Acknowledgements}

None.

\section{Footnote}

Conflicts of Interest: The authors have no conflicts of interest to declare.

\section{References}

1. Miller KD, Siegel RL, Lin CC, et al. Cancer treatment and survivorship statistics, 2016. CA Cancer J Clin 2016;66:271-89.

2. Hong D, Fritz AJ, Zaidi SK, et al. Epithelial-tomesenchymal transition and cancer stem cells contribute to breast cancer heterogeneity. J Cell Physiol 2018;233:9136-44.

3. Donovan D. Metastatic breast cancer epidemiology and management with a focus on taxanes. Clin J Oncol Nurs 2013;17 Suppl:5-8.

4. Hess G, Barlev A, Chung K, et al. Cost of palliative radiation to the bone for patients with bone metastases secondary to breast or prostate cancer. Radiat Oncol 2012;7:168.

5. Blanchette-Farra N, Kita D, Konstorum A, et al.
Contribution of three-dimensional architecture and tumor-associated fibroblasts to hepcidin regulation in breast cancer. Oncogene 2018;37:4013-32.

6. Ruchala P, Nemeth E. The pathophysiology and pharmacology of hepcidin. Trends Pharmacol Sci 2014;35:155-61.

7. Pinnix ZK, Miller LD, Wang W, et al. Ferroportin and iron regulation in breast cancer progression and prognosis. Sci Transl Med 2010;2:43ra56.

8. Bonfiglio R, Scimeca M, Toschi N, et al. Radiological, Histological and Chemical Analysis of Breast Microcalcifications: Diagnostic Value and Biological Significance. J Mammary Gland Biol Neoplasia 2018;23:89.

9. Scimeca M, Bonfiglio R, Montanaro M, et al. Osteoblastlike cells in human cancers: new cell type and reliable markers for bone metastasis. Future Oncol 2018;14:9-11.

10. Katagiri T, Watabe T. Bone Morphogenetic Proteins. Cold Spring Harb Perspect Biol 2016;8(6).

11. Scimeca M, Salustri A, Bonanno E, et al. Impairment of PTX3 expression in osteoblasts: a key element for osteoporosis. Cell Death Dis 2017;8:e3125.

12. Tarantino U, Feola M, Celi M, et al. PTX3: a new mediator of bone metabolism and osteoporosis. Muscles Ligaments Tendons J 2017;7:200-201.

13. Scimeca $M$, Antonacci $C$, Toschi N, et al. Breast Osteoblast-like Cells: A Reliable Early Marker for Bone Metastases From Breast Cancer. Clin Breast Cancer 2018;18:e659-69.

14. Li GF, Xu YJ, He YF, et al. Effect of hepcidin on intracellular calcium in human osteoblasts. Mol Cell Biochem 2012;366:169-74.

15. Lu H, Lian L, Shi D, et al. Hepcidin promotes osteogenic differentiation through the bone morphogenetic protein 2/small mothers against decapentaplegic and mitogenactivated protein kinase/P38 signaling pathways in mesenchymal stem cells. Mol Med Rep 2015;11:143-50.

16. Torti SV, Torti FM. Cellular iron metabolism in prognosis and therapy of breast cancer. Crit Rev Oncog 2013;18:435-48.

17. Bruehlmeier M, Leenders KL, Vontobel P, et al. Increased cerebral iron uptake in Wilson's disease: A 52Fe-citrate PET study. J Nucl Med 2000;41:781-7.

18. Bartnicka JJ, Blower PJ. Insights into Trace Metal Metabolism in Health and Disease from PET: "PET Metallomics". J Nucl Med 2018;59:1355-9.

19. Scimeca M, Bischetti S, Lamsira HK, et al. Energy Dispersive X-ray (EDX) microanalysis: A powerful tool 
Page 4 of 4

in biomedical research and diagnosis. Eur J Histochem 2018;62:2841.

20. Scimeca M, Orlandi A, Terrenato I, et al. Assessment of metal contaminants in non-small cell lung cancer by EDX microanalysis. Eur J Histochem 2014;58:2403.

Cite this article as: Scimeca M, Bonanno E. New highlight in breast cancer development: the key role of hepcidin and iron metabolism. Ann Transl Med 2018;6(Suppl 1):S56. doi: 10.21037/ atm.2018.10.30
Scimeca and Bonanno. Hepcidin and iron in breast cancer

21. Scimeca M, Urbano N, Bonfiglio R, et al. Management of oncological patients in the digital era: anatomic pathology and nuclear medicine teamwork. Future Oncol 2018;14:1013-5. 